\title{
Peritoneal dialysis infections: An opportunity for improvement
}

\author{
Anabela Rodrigues MD, PhD ${ }^{a}$, Marília Maciel PhD ${ }^{\mathrm{b}, \mathrm{c}}$, Cledir Santos $\mathrm{PhD}^{\mathrm{b}}$, \\ Diana Machado MS ${ }^{\mathrm{b}}$, Joana Sampaio MS ${ }^{\mathrm{b}}$, Nelson Lima $\mathrm{PhD}^{\mathrm{b}}$, Maria J. Carvalho MD ${ }^{\mathrm{a}}$, \\ António Cabrita $\mathrm{MD}^{\mathrm{a}}$, Margarida Martins $\mathrm{PhD}^{\mathrm{b}, *, \dagger}$ \\ ${ }^{a}$ Nephrology Department, Centro Hospitalar do Porto-Hospital Santo Antonio, Instituto de Ciências Biomédicas Abel Salazar and Unit for \\ Multidisciplinary Investigation in Biomedicine, University of Porto, Porto, Portugal \\ ${ }^{\mathrm{b}}$ Center of Biological Engineering, University of Minho, Braga, Portugal \\ ${ }^{\mathrm{c}}$ Mycology Department, Federal University of Pernambuco, Cidade Universitária, Recife, Pernambuco, Brazil
}

\section{Key Words:}

Transfer set

Microbiology

Gram-negative

Renal replacement therapy
Peritoneal dialysis (PD) catheter-associated infections remain a challenging cause of technique failure. Patient training and preventive measures are key elements in the management of infection rates. Twenty-seven of the 167 PD catheter transfer sets analyzed (19\%) yielded a positive microbial culture (58\% gram-negative bacteria). These results show that subclinical contamination, particularly from environmental gram-negative bacteria, is a potential hazard, indicating the need for a protocol for regular transfer set changes.

Copyright $\odot 2014$ by the Association for Professionals in Infection Control and Epidemiology, Inc. Published by Elsevier Inc. All rights reserved.
Peritoneal dialysis (PD) is gaining increasing acceptance as a renal replacement therapy owing to its economic and social benefits. Despite the technical advances in PD, peritonitis remains a major cause of technique failure. ${ }^{1,2}$ Microorganisms causing PDrelated infections can originate from several sources. ${ }^{3}$ Skin and environmental microorganism contamination associated with transfer set manipulation are considered the major causes of infection. ${ }^{2}$ Thus, the success of PD therapy relies on patient skills and compliance with the personalized education programs to minimize contamination. ${ }^{4}$ However, patients often disregard care protocols, including hand hygiene. ${ }^{5}$ Furthermore, infection prevention measures empirically include a routine transfer set change

\footnotetext{
* Address correspondence to Margarida Martins, PhD, Anabela Rodrigues Nephrology Department Hospital Geral de Santo António Largo Professor Abel Salazar, 4099-001 Porto, Portugal.

E-mail address: martins.margarida@gmail.com (M. Martins).

In memoriam: We will always keep in memory Rosário Oliveira ${ }^{\mathrm{b}}$, her contributions and enthusiasm as an exceptional scientist and true friend, which have been stimulating for all of us.

M.M. is supported by the Portuguese Foundation for Science and Technology (contract SFRH/BPD/73663/2010), financed by the European Social Fund and national funds from the Ministério da Educação e Ciência. This work was supported by a research grant from the Portuguese Society of Nephrology (to A.R.) and the Portuguese Foundation for Science and Technology Strategic Project PEst-OE/EQB/ LA0023/2013.

Conflict of interest: None to report.

Present address: 3B's Research Group-Biomaterials, Biodegradables and Biomimetics, Department of Polymer Engineering, University of Minho; Headquarters of the European Institute of Excellence on Tissue Engineering and Regenerative Medicine; AvePark, Caldas das Taipas, Guimarães, Portugal.
}

every 6 months, based on the assumption that the longer the usage time, the more likely the segment will be colonized by environmental microorganisms.

In a single study of $17 \mathrm{PD}$ transfer sets, culturable microorganisms were not identified $^{6}$; however, our group has reported the presence of biofilm on PD transfer sets and identified their chemical constituents. ${ }^{3}$ Thus, the present study aimed to evaluate the microbiological spectrum and density of microorganisms on the intraluminal surface of transfer sets, to elucidate the relevance of this catheter segment as a port of entry for microorganisms causing infections.

\section{MATERIALS AND METHODS}

Between September 2011 and June 2013, transfer sets were obtained from adults undergoing PD at the Division of Nephrology, Hospital Geral de Santo António. All of the transfer sets were removed within the routine clinical time frame (after 6 months of use). Exclusion criteria included removal owing to suspected fault/crack, malfunction, touch contamination, and dropout. The International Society for Peritoneal Dialysis 2010 PD-related infections criteria were applied. ${ }^{7}$ This study was approved by the Ethics Committee of Centro Hospitalar do Porto [PI-243/11 (153-DEFI/229-CES)], and all patients provided signed consent.

The transfer set outer surface was first disinfected by immersion in $70 \%$ ethanol for 10 minutes, followed by a rinse and immersion in sterile water for 5 minutes, and wiping with sterile gauze. The ends of the transfer set were cut, and the segment was split longitudinally. The biofilm ${ }^{3}$ was scrapped and suspended in $400 \mu \mathrm{L}$ of saline, then sonicated at $50-60 \mathrm{~Hz}$ (Sonicor, NY) for 5 minutes and 


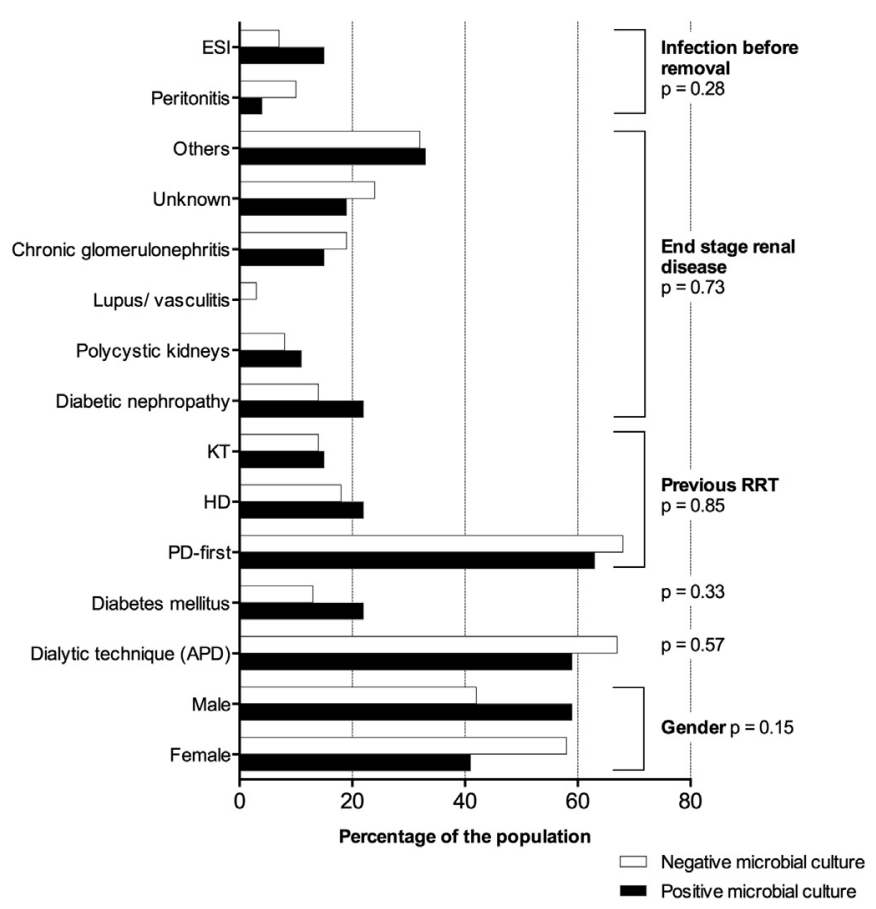

Fig 1. Population characteristics according to the microbial recovery outcome. Categorical variables were compared using the $\chi^{2}$ test, with Yates correction applied when required. $A P D$, automated PD; ESI, exit site infection; $H D$, hemodialysis; $K T$, kidney transplant; $R R T$, renal replacement therapy.

vortexed for 30 seconds. This protocol preserves microbial culturability. ${ }^{8}$

For semiquantitative analysis, $100 \mu \mathrm{L}$ of the sonicated suspension was plated onto Oxoid Tryptose Blood Agar Base (Thermo Scientific, Waltham, MA) supplemented with 7\% defibrinated sheep's blood (Probiologica, Lisboa, Portugal) (TBA) and then incubated at $37^{\circ} \mathrm{C}$ for up to 72 hours. For qualitative analysis, the residual suspension was inoculated in $5 \mathrm{~mL}$ of Tryptic Soy Broth (Liofilchem, Teramo, Italy) supplemented with $7 \%$ defibrinated sheep's blood and incubated at $37^{\circ} \mathrm{C}$ under $120 \mathrm{rpm}$ spinning for 24 hours, followed by subculturing on TBA at $37^{\circ} \mathrm{C}$ for up to 72 hours. All plates were examined for microbial growth, and the number of colony-forming units (CFU) was recorded. The microbial isolates were identified by matrix-assisted laser desorption/ionization time-of-flight intact cell mass spectrometry (Axima LNR system; Shimadzu Biotech, Kyoto, Japan). ${ }^{9}$

\section{RESULTS}

A total of 167 transfer sets were removed from 82 patients. The samples were divided in 2 groups according to the presence $(\mathrm{n}=27$ [19\%]; mean age, $49.5 \pm 13$ years) or absence $(n=140$ [81\%]; mean age, $51.9 \pm 15.2$ years) of microorganisms by semiquantitative and qualitative culture. The population characteristics were similar in the 2 study groups (Fig 1); in particular, rates of peritonitis and exit site infection before removal were $4 \%$ and $15 \%$, respectively, in those with a positive microbial culture and $10 \%$ and $7 \%$ in those with a negative microbial culture $(P=.28)$.

In the 27 samples with a positive culture, $41 \%$ had $\leq 8 \mathrm{CFU} /$ transfer set, $44 \%$ had $9 \leq \mathrm{CFU} /$ transfer set $<9 \times 10^{2}$, and only $15 \%$ yielded $9 \times 10^{2} \leq \mathrm{CFU} /$ transfer set $<9 \times 10^{4}$. Gram-negative bacteria were more commonly isolated than gram-positive bacteria ( $58 \%$ vs $39 \%$ ). Sphingomonas spp were the most common microorganisms (27\%), followed by coagulase-negative Staphylococcus (24\%). No
Table 1

Microbial growth in transfer sets with a positive culture

\begin{tabular}{lcc}
\hline Species & $\mathrm{n}(\%)^{*}$ & ${\text { Score, } \text { median }^{\dagger}}^{\dagger}$ \\
\hline Gram-positive & $13(39)$ & $\mathbf{1}$ \\
Staphylococcus epidermidis & $5(15)$ & 1 \\
Micrococcus luteus & $4(12)$ & 1 \\
Staphylococcus capitis & $2(6)$ & 2 \\
Staphylococcus aureus & $1(3)$ & 2 \\
Staphylococcus warneri & $1(3)$ & 3 \\
Gram-negative & $19(58)$ & $\mathbf{2}$ \\
Sphingomonas spp & $9(27)$ & 2 \\
Delftia acidovorans & $3(9)$ & 1 \\
Herbaspirillum huttiense & $3(9)$ & 2 \\
Burkholderia spp. & $2(6)$ & 1.5 \\
Pseudomonas aeruginosa & $1(3)$ & 2 \\
Serratia marcescens & $1(3)$ & 3 \\
Fungi & $1(3)$ & $\mathbf{2}$ \\
Pichia guilliermondii & $1(3)$ & 2 \\
\hline
\end{tabular}

The median score values for each main group are shown in bold.

*Six of 27 samples had more than 1 bacterial species, resulting in a total of 33 cultured microorganisms.

†The combined results from the qualitative and semiquantitative analyses were classified into 3 categories: 1 , growth in the qualitative analysis and $\leq 8 \mathrm{CFU} /$ transfer set in the semiquantitative analysis; $2,9 \leq \mathrm{CFU} /$ transfer set $<9 \times 10^{2} ; 3$, $9 \times 10^{2} \leq \mathrm{CFU} /$ transfer set $<9 \times 10^{4}$. The detection limit of the semiquantitative method was $8 \mathrm{CFU} /$ transfer set.

association was observed between microbial density and microbial gram positivity or negativity $(P=.58)$.

The consecutive recovery of microorganisms from transfer sets belonging to the same patient was rare $(n=2)$, and different microorganisms were recovered at the different time points (case 1: Delftia acidovorans and Burkholderia spp; case 2: Herbaspirillum huttiense and Pichia guilliermondii).

In addition, microorganisms were not recovered from the transfer set in the majority of situations when an infection episode occurred before segment removal (Fig 1). In the 5 samples in which growth was observed, the microorganism isolated from the transfer set was not that causing the infection (Sphingomonas spp vs Providencia rettgeri, H huttiense vs Staphylococcus aureus, Staphylococcus capitis vs $S$ aureus, Sphingomonas spp and D acidovorans vs Streptococcus spp, and Micrococcus luteus vs Kocuria kristinae).

\section{DISCUSSION}

This study involved a single center running a PD program representative of the modality in Portugal. The rate of peritonitis in this center between 2006-2010 was 0.47 episodes/patient-year (ep/ $\mathrm{p}-\mathrm{y}$ ), including 0.13 gram-negative ep/p-y and 0.22 gram-positive ep/p-y peritonitis (0.05 Staphylococcus epidermidis ep/p-y). ${ }^{1}$ The low incidence of microbial recovery in the transfer sets (Fig 1) is undoubtedly related to patient training and infection prevention protocols; nonetheless, our findings show that contamination of transfer sets by gram-negative species represents a potential hazard.

Environmental contamination of segments is suggested by the low microbial burden (Table 1 ). It should be noted that the present study did not assess the intraluminal migration of microorganisms; however, touch contaminations have been associated with low burden of microorganisms entering the peritoneal cavity ${ }^{10}$ and low peritonitis rates, ${ }^{11}$ possibly related to the effect of the flush-before-fill procedure that helps "wash" the lumen. Furthermore, the microbial spectrum (Table 1), along with the general lack of consistent microbial recovery and/or species identity at different sampling time points, also differing from previously identified infectious agents, point to an environmental source. In fact, skin or mouth colonizers such as Staphylococcus spp and Micrococcus spp, were observed (Table 1). Importantly, a high rate of gram-negative bacteria associated with water and moist environments, including Sphingomonas spp and 
Burkholderia spp, was detected (Table 1). These microorganisms have been associated with serious infectious episodes in the literature, although the outcome of an infection depends not only on the microorganism, but also on patient morbidity and dialysis factors. This is particularly relevant given the increasing rate of gram-negative infections, which are associated with poor outcomes. ${ }^{2,7}$ Furthermore, whereas touch contamination is usually associated with grampositive bacteria, this study suggests that potentially hazardous gram-negative microorganisms present, for example, on work surfaces, on hands, and in watery environments can gain access to the PD catheter through touch during transfer set manipulation.

Overall, this study demonstrates that the PD catheter transfer set can serve as a port of entry for infectious microorganisms, and reinforces the relevance of transfer set removal as an infection preventive measure. Although the rate of coagulase-negative Staphylococcus peritonitis has been suggested as an indication of adequate training, ${ }^{4}$ our results point to the need for improvements in patient training measures to limit environmental gram-negative bacteria contamination.

\section{Acknowledgment}

We thank Olívia Santos, Ana Abrunhosa, and Cristiana Sarmento for sample collection.

\section{References}

1. Rocha A, Rodrigues A, Teixeira L, Carvalho MJ, Mendonça D, Cabrita A. Temporal trends in peritonitis rates, microbiology and outcomes: the major clinical complication of peritoneal dialysis. Blood Purif 2012;33:284-91.

2. Piraino B, Bernardini J, Brown E, Figueiredo A, Johnson DW, Lye WC, et al. ISPD position statement on reducing the risks of peritoneal dialysis-related infections. Perit Dial Int 2011;31:614-30.

3. Martins M, Rodrigues A, Pedrosa JM, Carvalho MJ, Cabrita A, Oliveira R. Update on the challenging role of biofilms in peritoneal dialysis. Biofouling 2013;29: 1015-27.

4. Piraino B. Today's approaches to prevent peritonitis. Contrib Nephrol 2012; 178:246-50.

5. Firanek C, Guest S. Hand hygiene in peritoneal dialysis. Perit Dial Int 2011;31: 399-408.

6. Verger C, Chesneau AM, Thibault M, Bataille N. Biofilm on Tenckhoff catheters: a possible source for peritonitis. Perit Dial Int 1987;7:174-8.

7. Li PK, Szeto CC, Piraino B, Bernardini J, Figueiredo AE, Gupta A, et al. Peritoneal dialysis-related infections recommendations: 2010 update. Perit Dial Int 2010; 30:393-423.

8. Trampuz A, Piper KE, Jacobson MJ, Hanssen AD, Unni KK, Osmon DR, et al. Sonication of removed hip and knee prostheses for diagnosis of infection. N Engl J Med 2007;357:654-63.

9. Santos C, Lima N, Sampaio P, Pais C. Matrix-assisted laser desorption/ionization time-of-flight intact cell mass spectrometry to detect emerging pathogenic Candida species. Diagn Microbiol Infect Dis 2011:71:304-8.

10. Miller TE, Findon G. Touch contamination of connection devices in peritoneal dialysis: a quantitative microbiologic analysis. Perit Dial Int 1997;17: 560-7.

11. Silverstein DM, Wilcox JE. Outcome of accidental peritoneal dialysis catheter holes or tip exposure. Pediatr Nephrol 2010;25:1147-51. 